\title{
Network Pharmacology-Based Systematic Analysis of Molecular Mechanisms of Geranium wilfordii Maxim for HSV-2 Infection
}

\author{
Hao Zhang $\left(\mathbb{D}\right.$, Ming-Huang Gao $\mathbb{D}^{D}$, Yang Chen $(\mathbb{D}$, and Tao Liu $(\mathbb{D}$ \\ Department of Integrated Traditional Chinese and Western Medicine, Nanjing University of Chinese Medicine, \\ Nanjing 210000, China \\ Correspondence should be addressed to Tao Liu; liutao@njucm.edu.cn
}

Received 29 May 2021; Revised 3 October 2021; Accepted 13 October 2021; Published 3 November 2021

Academic Editor: Oluwafemi Adeleke Ojo

Copyright (c) 2021 Hao Zhang et al. This is an open access article distributed under the Creative Commons Attribution License, which permits unrestricted use, distribution, and reproduction in any medium, provided the original work is properly cited.

Background. Being a traditional Chinese medicine, Geranium wilfordii Maxim (GWM) is used for the treatment of various infectious diseases, and its main active ingredients are the polyphenolic substances such as polyphenols quercetin, corilagin, and geraniin. Previous studies have demonstrated the anti-HSV-1 viral activity of these three main ingredients. Through employing a network pharmacological method, the authors of the present research intend to probe the mechanism of GWM for the therapeutic treatment of HSV-2 infection. Methods. The bioactive substances and related targets of GWM were obtained from the TCMSP database. Gene expression discrepancy for HSV-2 infection was obtained from dataset GSE18527. Crossover genes between disease target genes and GWM target genes were gained via Circos package. Distinctively displayed genes (DDGs) during HSV-2 infection were uploaded to the Metascape database with GWM target genes for further analysis. The tissue-specific distribution of the genes was obtained by uploading the genes to the PaGenBase database. Ingredient-gene-pathway (IGP) networks were constructed using Cytoscape software. Molecular docking investigations were carried out utilizing AutoDock Vina software. Results. Nine actively involved components were retrieved from the TCMSP database. After taking the intersection among 153 drug target genes and 83 DDGs, 7 crossover genes were screened. Gene enrichment analysis showed that GWM treatment of HSV2 infection mainly involves cytokine signaling in the immune system, response to virus, epithelial cell differentiation, and type II interferon signaling (IFNG). One hub, three core objectives, and two critical paths were filtered out from the built network. Geraniin showed strong binding activity with HSV-2 gD protein and STING protein in molecular docking. Conclusions. This network pharmacological study provides a fundamental molecular mechanistic exploration of GWM for the treatment of HSV2 infection.

\section{Introduction}

Genital herpes is a common sexually transmitted infection (STI) caused by herpes simplex virus type 2 (HSV-2) and represents a major health problem globally [1].

HSV-2 frequently modulates the cytokine milieu of the microenvironment in favor of HIV-1 spread [2]. The available antiviral agents used in HSV-2 infections are those that are clinically approved for the general treatment of HSV-2 infections, such as acyclovir and famciclovir. Indeed, previous studies of HSV-2 infection indicated that the use of single nucleoside analogues is inadequate for effective control of virus replication, as the administered nucleoside analogues often exert significant selection pressure on the virus, leading to the rapid generation of escape mutants. While current therapies based on nucleoside analogues suppress viral replication and reduce progression of HSV-2 infection, treatment is lifelong and viral cure is extremely rare [3]. Therefore, to further optimize treatment, new effective drugs are highly warranted.

GWM is a traditional Chinese medicine, and it contains geraniin, quercetin, corilagin, and so on [4]. Previous studies have demonstrated the anti-HSV-1 viral activity of these three main ingredients [5-7]. One study revealed a promising role of geraniin as an antiviral agent against HSV-2 infection with no apparent toxicity [8]. However, the mechanisms by which GWM inhibits HSV-2 infection remain unclear. 
Traditional Chinese medicine (TCM) has the characteristics of multitarget, multistep, and multilevel synergism [9]. Recently, network pharmacology becomes an important bioinformatics tool for identifying the mechanism of action of TCM [10]. In the present study, the network pharmacology approach was performed to further investigate the active ingredients and the underlying mechanism of GWM for the treatment of HSV-2. A flow diagram summarizing the different procedures of this study is shown in Figure 1.

\section{Materials and Methods}

2.1. Bioactive Chemical Substance and Objective Genes of GWM. Bioactive components and action targets of GWM were screened in the TCMSP website (old.tcmsp-e.com/ tcmsp.php) [11]. Oral bioavailability (OB) $\geq 30 \%$ and druglike properties $(\mathrm{DL}) \geq 0.18$ were the filtering criteria [12]. The active components were filtered using pharmacokinetic absorption, distribution, metabolism, and excretion guidelines (ADME) filter. Since the polyphenolic substance corilagin and geraniin are the main active ingredients of GWM, they are also listed. The active ingredient target genes were normalized in the UniProt database (uniprot.org). The structures of the active compounds were acquired at PubChem website (pubchem.ncbi.nlm.nih.gov).

2.2. DDGs in HSV-2 Infection. The GSE18527 dataset in the GEO database (ncbi.nlm.nih.gov/geo) was created by Peng T et al. The dataset had 19 samples, consisting of 3 cases of pretreatment healthy skin, 4 cases of pretreatment diseased skin, 6 cases of diseased skin in the healing group, and 6 cases of healthy skin in the healing group. The screening criteria for distinctively displayed genes were $P<0.05$ and $\operatorname{logFC}>4$. The DDGs were established by the intersection of the two datasets group: pretreatment healthy skin and pretreatment lesioned skin group and posttreatment diseased skin and pretreatment lesioned skin group.

2.3. Intersect Target Genes. Crossover genes between DDGs and GWM target genes were obtained using Circos software.

2.4. Gene Pathway and Functional Enrichment Analysis. DDGs during HSV-2 infection and GWM target genes were uploaded to the Metascape database (metascape.org) for further analysis of relevant genes and functional enrichment.

2.5. Histospecific Gene Enrichment Analysis. The distribution of genes was further analyzed after uploading DDGs during HSV-2 infection and GWM target genes to the PaGenBase database (bioinf.xmu.edu.cn/PaGenBase).

2.6. Enrichment Analysis of Transcription Factor Targets. To assess the potential regulatory patterns of the most enriched and conserved transcripts, the DDGs during HSV2 infection and GWM target genes were submitted to transcription factor (TF) enrichment analysis by using the TRANSFAC Predicted Transcription Factor Targets dataset
(https://maayanlab.cloud/Harmonizome/dataset/

TRANSFAC + Predicted + Transcription + Factor + Targets). The obtained TFs were sorted according to their average enrichment scores. The top 20 TFs of both sets of mRNAs were further evaluated to determine their coregulatory network.

2.7. Ingredient-Gene-Pathway (IGP) Network. The IGP network was created by importing five intersecting active ingredients, seven intersecting genes, and the leading 17 KEGG pathways into Cytoscape software. The topological parameters such as degree centrality (DC), betweenness centrality (BC), and closeness centrality (CC) were utilized to evaluate the centrality features of nodes in IGP networks.

2.8. Molecular Docking Studies. The HSV-2 gD protein, STING protein, and drug structures were downloaded from the PDB (pdb.org) and PubChem websites. Drug and HSV-2 gD protein and STING protein docking investigations were conducted in AutoDock Vina (version 1.1.2) [13]. The visualization of the docking performance was performed by PyMOL v.2.3 software, and the docking effect was evaluated using the affinity value (AV).

\section{Results}

3.1. Bioactive Components and Drug Targets of GWM. Nine GWM bioactive chemicals were obtained in the TCMSP database, containing ellagic acid, sitosterol, kaempferol, furosin, ethyl brevifolincarboxylate, luteolin, quercetin, dehydrogeraniin, and corilagin. We downloaded the two-dimensional structure of the chemicals in PubChem website (Table 1). 309 drug targets were acquired in the TCMSP website and converted to target genes in the UniProt website. 153 target genes were isolated as drug-targeting genes after deleting repetitions.

3.2. DDGs in HSV-2 Infection. Finally, 40 genes upregulated and 43 genes downregulated were selected. The Venn diagram and heat map of DDGs are shown in Figure 2.

3.3. Intersect Target Genes. Seven crossover genes (CXCL10, CXCL11, CXCL8, IL-6 and IL-1 $\beta$, MMP1, and SELE) were filtered in GWM target genes and HSV-2 DDGs via Circos package, as shown in Figure 3.

3.4. Gene Pathway and Function Enrichment Analysis. The enrichment analysis of GWM target genes and DDGs during HSV-2 infection were jointly clustered in 26 enrichment items, and the most significant ones included cytokine signaling in the immune system, response to virus, epithelial cell differentiation, and type II interferon signaling (IFNG), as shown in Figure 4. These analyses suggested that GWM may treat HSV-2 infection by modulating cytokine signaling in the immune system, the process of cell state or activity changes due to viral stimulation, and the process by which unspecialized cells acquire specialized features of 


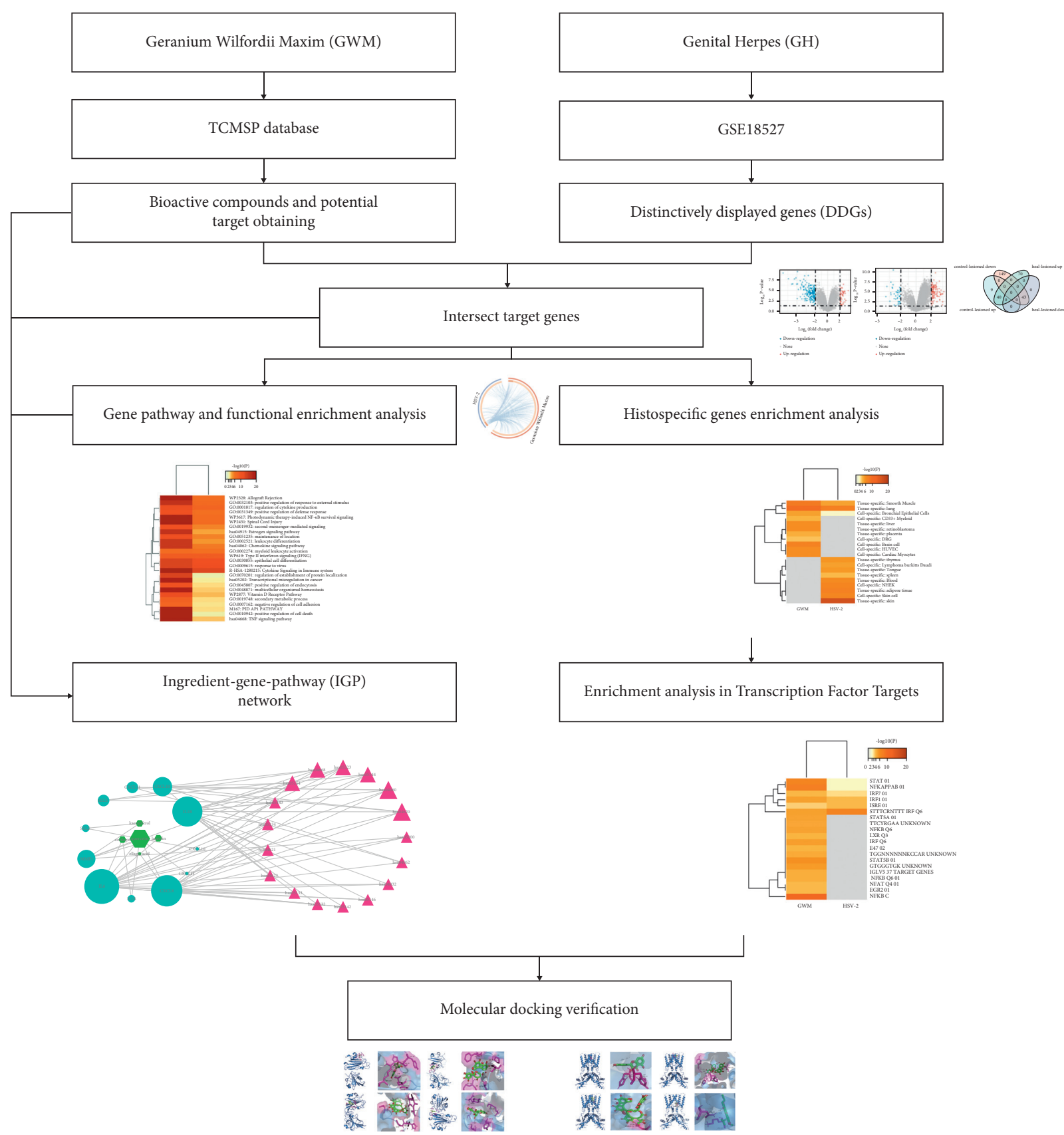

Figure 1: Workflow chart of Geranium wilfordii Maxim in the treatment of genital herpes based on network pharmacology.

epithelial cells, the binding of IFNG to its receptor, and the subsequent phosphorylation cascade reaction involving the JAK and STAT protein families.

3.5. Histospecific Gene Enrichment Analysis. PaGenBase database tissue-specific enrichment profiling indicated that the target genes of GWM were mainly concentrated in lung and smooth muscle tissues; cell-specific was brain cell. The DDGs of HSV-2 were mainly enriched in skin tissue, followed by lung and smooth muscle tissues. Cell-specific was NHEK (normal human epidermal keratinocytes), as shown in Figure 5.
3.6. Enrichment Analysis in Transcription Factor Targets. Enrichment analysis of GWM target genes and HSV-2 DDGs transcription factors focused on STTTCRNTTT IRF Q6, ISRE 01, IRF1 01, IRF7 01, NFKAPPAB 01, and STAT 01, as shown in Figure 6. This suggested that GWM may regulate the ability of target genes during the progression of HSV-2 infection through changes in the activity or expression of the above transcription factors, thereby controlling HSV-2 infection.

3.7. Ingredient-Gene-Pathway (IGP) Network. The IGP network consists of 29 nodes (5 active ingredients, 7 intersecting genes, and 17 pathways) and 73 edges. In this 
TABLE 1: Active ingredients and ADME parameters of Geranium wilfordii Maxim (GWM).

\begin{tabular}{|c|c|c|c|c|c|c|c|}
\hline NO. & Molecule ID & Molecule name & Chemical formula & Structure & MW & OB (\%) & $\mathrm{DL}$ \\
\hline 1 & MOL001002 & Ellagic acid & $\mathrm{C}_{14} \mathrm{H}_{6} \mathrm{O}_{8}$ & & 302.2 & 43.06 & 0.43 \\
\hline 2 & MOL000359 & Sitosterol & $\mathrm{C}_{29} \mathrm{H}_{50} \mathrm{O}$ & & 414.79 & 36.91 & 0.75 \\
\hline 3 & MOL000422 & Kaempferol & $\mathrm{C}_{15} \mathrm{H}_{10} \mathrm{O}_{6}$ & & 286.25 & 41.88 & 0.24 \\
\hline 4 & MOL005067 & Furosin & $\mathrm{C}_{27} \mathrm{H}_{22} \mathrm{O}_{19}$ & & 650.49 & 40.53 & 0.29 \\
\hline 5 & MOL005073 & Ethyl brevifolin carboxylate & $\mathrm{C}_{15} \mathrm{H}_{12} \mathrm{O}_{8}$ & & 320.27 & 30.86 & 0.33 \\
\hline 6 & MOL000006 & Luteolin & $\mathrm{C}_{15} \mathrm{H}_{10} \mathrm{O}_{6}$ & & 286.25 & 36.16 & 0.25 \\
\hline 7 & MOL000098 & Quercetin & $\mathrm{C}_{15} \mathrm{H}_{10} \mathrm{O}_{7}$ & & 302.25 & 46.43 & 0.28 \\
\hline 8 & MOL005064 & Dehydrogeraniin & $\mathrm{C}_{41} \mathrm{H}_{28} \mathrm{O}_{28}$ & & 968.68 & 59.57 & 0.01 \\
\hline 9 & MOL005079 & Corilagin & $\mathrm{C}_{27} \mathrm{H}_{22} \mathrm{O}_{18}$ & & 634.49 & 3.01 & 0.44 \\
\hline
\end{tabular}

ADME: absorption, distribution, metabolism, and excretion.

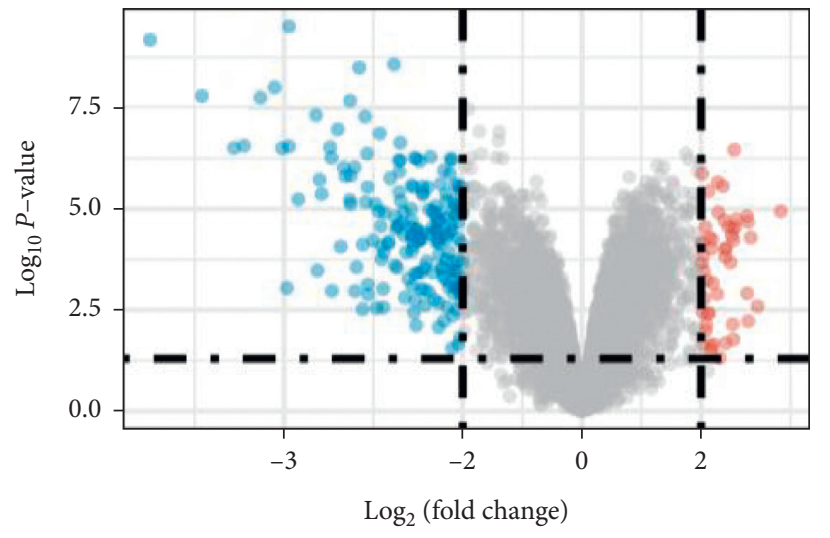

- Down-regulation

- None

- Up-regulation

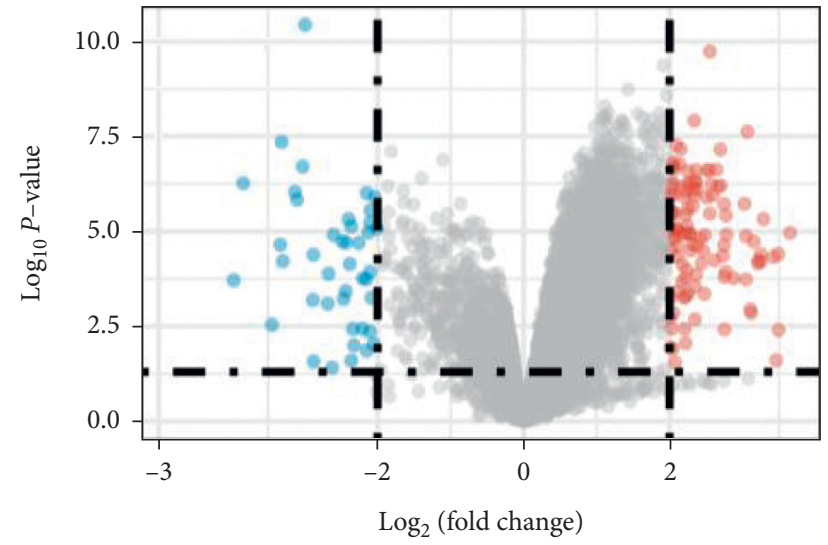

- Down-regulation

- None

- Up-regulation

(a)

FIgURE 2: Continued. 


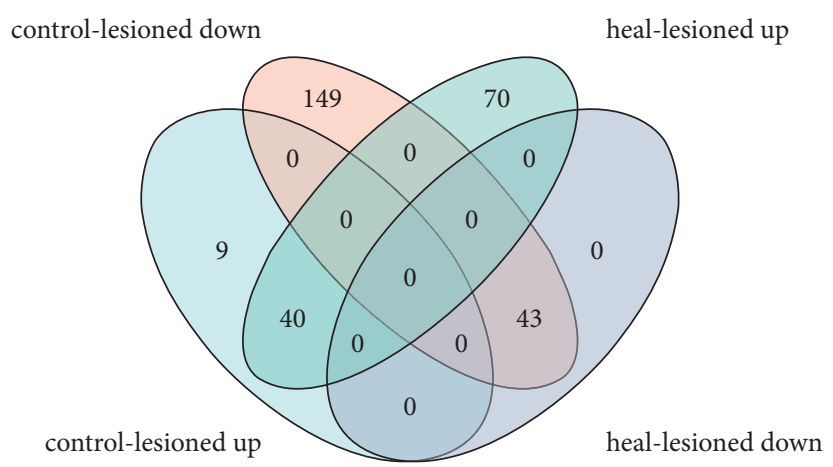

(c)

Figure 2: (a) Volcano map displays the differential genes between the pretreatment healthy skin and the pretreatment lesioned skin group. (b) Volcano map displays the differential genes between the posttreatment lesioned skin and the pretreatment lesioned skin group. (c) Venn diagram displays the intersection of the above 2 groups of differential genes. Up represents upregulated genes, and down represents downregulated genes.

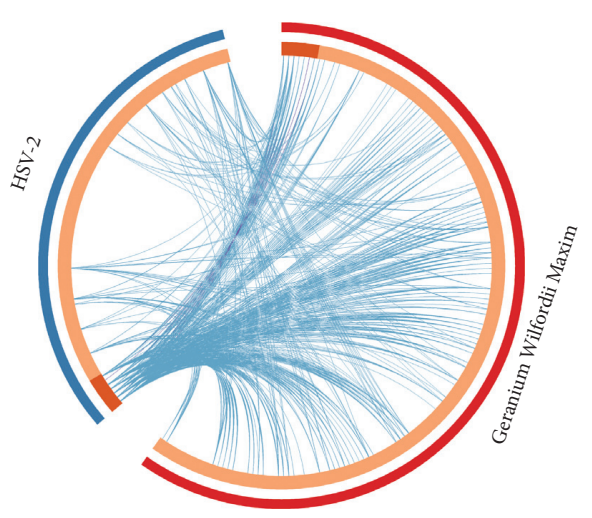

FIGURE 3: Red represents 153 gene targets of Geranium wilfordii Maxim; blue represents 83 genes significantly upregulated during HSV-2 infection; orange represents the intersection of the two groups of genes.

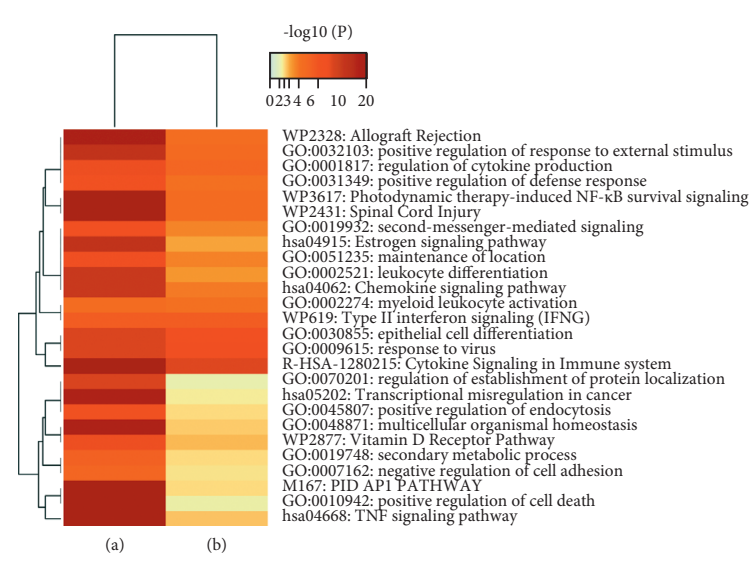

FIGURE 4: Gene pathway and functional enrichment analysis. (a) Enrichment analysis of 153 genes that are targets for the action of GWM. (b) Enrichment analysis of 83 genes that are significantly altered during HSV-2 infection.

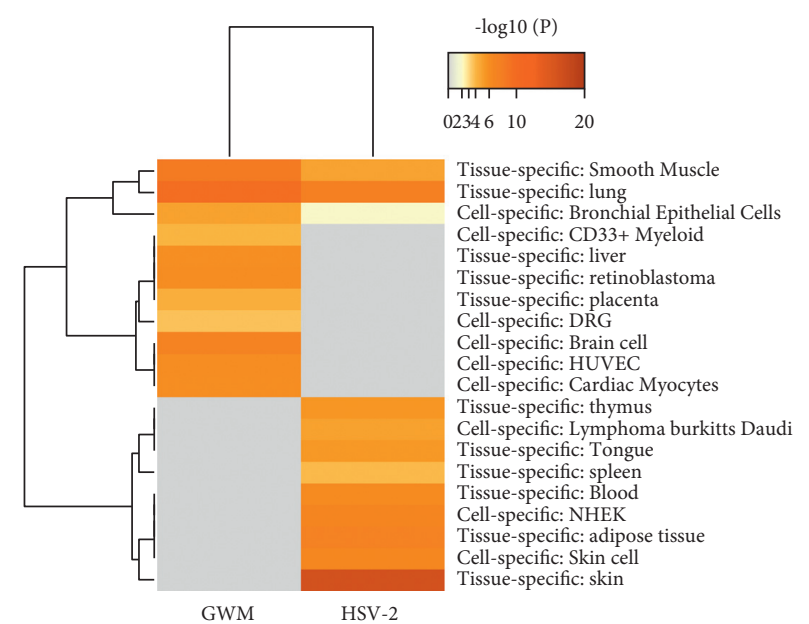

FIGURE 5: Summary of histospecific gene enrichment analysis in PaGenBase.

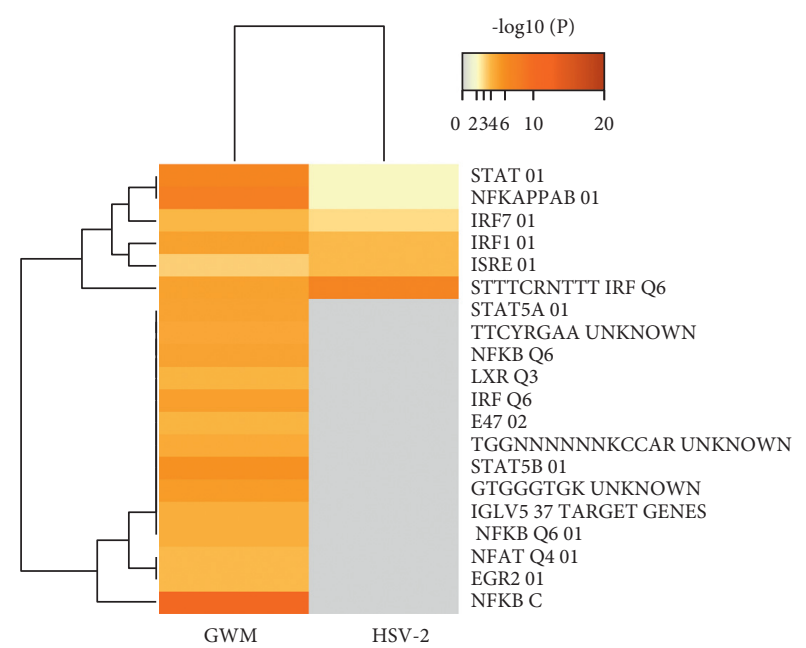

FIGURE 6: Summary of enrichment analysis in transcription factor targets. 


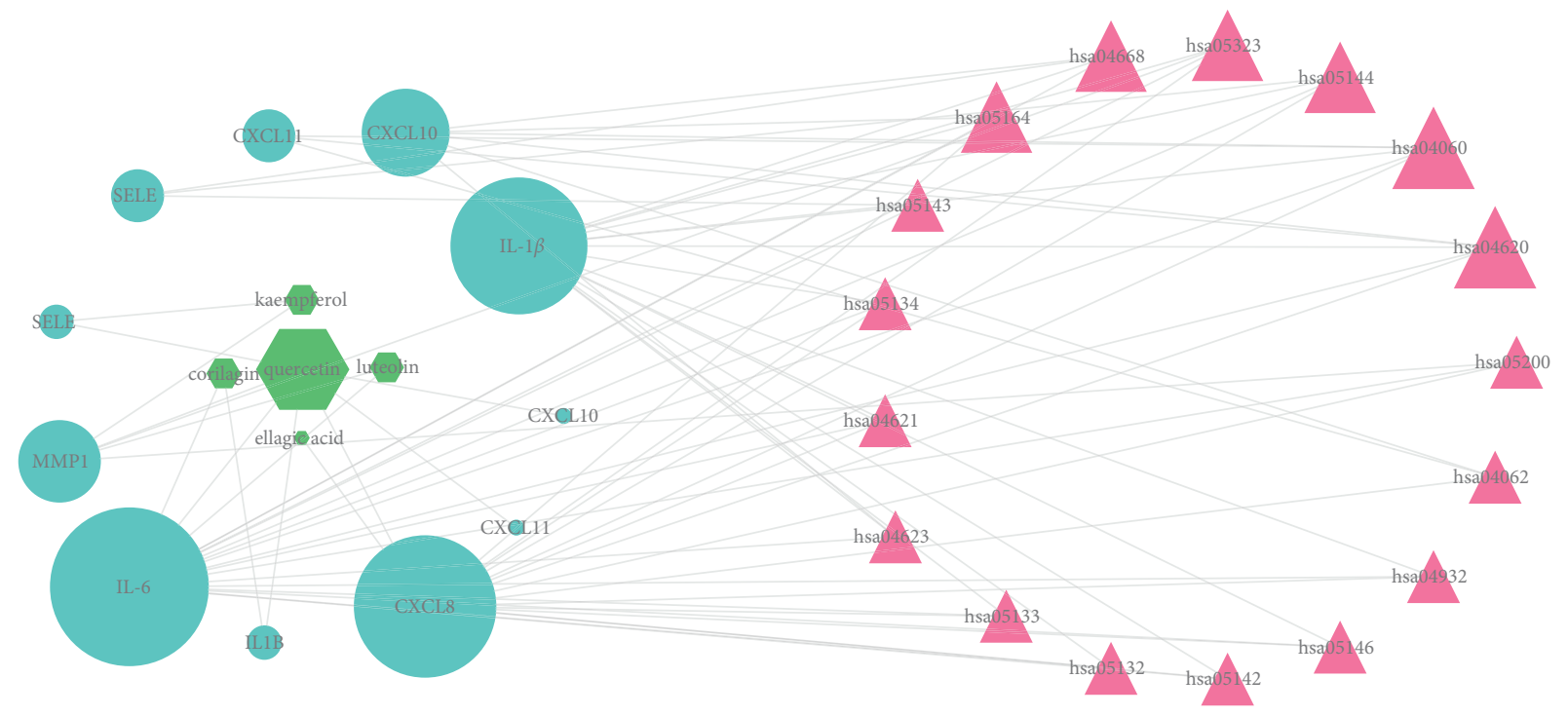

FIGURE 7: Ingredient-gene-pathway networks. Fuchsia triangles, aqua ellipses, and lime octagons stand for pathways of GWM, intersecting with target genes and active components, respectively. The bigger the shape of the graph, the larger the degree value of the node and the higher the role in the network.

network, we found that all 7 intersecting genes were related to quercetin, as shown in Figure 7. It is inferred that quercetin could be the pivotal effective component of GWM for the treatment of HSV-2 infection. According to the topological analysis, IL-6, IL- $1 \beta$, and CXCL8 are the pivotal genes. Toll-like receptor signaling pathway and cytokinecytokine receptor interaction pathway are the key pathways in the IGP network. Among them, IL-6, IL-1 $\beta$, and CXCL10 are associated with the cytosolic DNA-sensing pathway. Since IFN $\beta$ exerts a crucial function in the inhibition of HSV-2, HSV - 2 has evolved multiple means to inhibit IFN $\beta$ expression to produce immune escape [14-16], and the cGAS-STING pathway is a key mechanism for IFN $\beta$ production [17]; it is hypothesized that GWM may act through IL-6, IL- $1 \beta$, and CXCL10 in the cGAS-STING pathway as a key link in controlling HSV-2 infection.

3.8. Molecular Docking Studies. Nectin-1 is a cell adhesion protein, and binding of Nectin-1 protein by HSV-2 gD protein is necessary for HSV-2 to enter infected cells [18]. Epigallocatechin gallate (EGCG) has been shown to bind directly to HSV-2 gD protein to exert its anti-HSV-2 infection effect [19]. We docked quercetin, corilagin, geraniin, and EGCG to HSV-2 gD protein molecules respectively, and the binding activity of all the three was superior to that of EGCG, with geraniin showing the strongest binding activity $(-17.44 \mathrm{kcal} / \mathrm{mol})$, as shown in Table 2 . cGAS acts as the primary intracellular double-stranded DNA (dsDNA) sensor, sensing intracellular dsDNA and generating the secondary messenger cGMP-AMP (cGAMP), which is further sensed by the sensing protein STING downstream of the interferon gene, leading to IFN $\beta$ production [20]. HSV -2 has evolved multiple strategies to counteract this pathway, inhibiting IFN $\beta$ production and evading host immunity [21]. Mangostin is a STING-targeted pathway agonist [22].
TABLE 2: Docking scores of active ingredients of GWM with potential targets.

\begin{tabular}{lccc}
\hline Targets & PDB ID & Compound & Affinity $(\mathrm{kcal} / \mathrm{mol})$ \\
\hline HSV-2 gD & $4 \mathrm{MYV}$ & Quercetin & -7.92 \\
HSV-2 gD & $4 \mathrm{MYV}$ & Corilagin & -13.08 \\
HSV-2 gD & $4 \mathrm{MYV}$ & Geraniin & -17.44 \\
HSV-2 gD & $4 \mathrm{MYV}$ & EGCG & -6.88 \\
STING & $6 \mathrm{NT5}$ & Quercetin & -7.1 \\
STING & 6NT5 & Corilagin & -11.65 \\
STING & $6 \mathrm{NT5}$ & Geraniin & -11.71 \\
STING & $6 \mathrm{NT5}$ & Mangostin & -4.52 \\
\hline
\end{tabular}

The binding activity of all the three was superior to that of mangostin, with geraniin showing the strongest binding activity $(-11.71 \mathrm{kcal} / \mathrm{mol})$, as shown in Table 2 . These data suggested that quercetin, corilagin, and geraniin may affect the pathogenic process of HSV-2 by binding to the HSV-2 $\mathrm{gD}$ protein (Figure 8 ) interfering with the binding to the NECTIN receptor. The direct action of quercetin, corilagin, and geraniin with the cGAS-STING pathway relationship has yet to be experimentally verified. Molecular docking is shown in Figure 9.

\section{Discussion}

Five effective chemicals, namely, quercetin, corilagin, kaempferol, luteolin, and ellagic acid, were evaluated in the IGP network. Among them, quercetin exhibited the strongest node value, revealing that quercetin acts as a major element in the network. In a meta-analysis, quercetin-type flavonols were noted to have antiviral activity and significantly reduced the mortality of infected animals [23]. Three central genes were screened in the IGP network, including IL-6, IL-1 $\beta$, and CXCL8, which were all linked to quercetin. We hypothesized that GWM may exert antiviral effects by regulating the above targets during HSV-2 infection. 

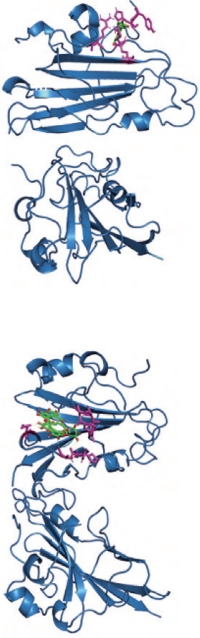

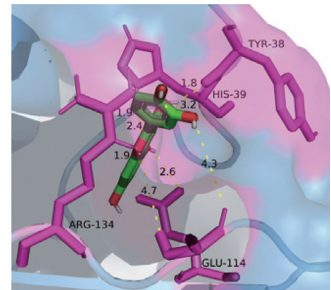

(a)

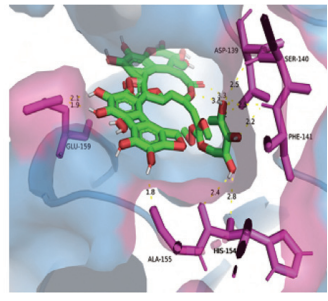

(c)
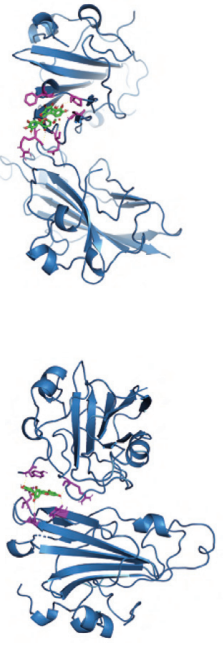

(d)

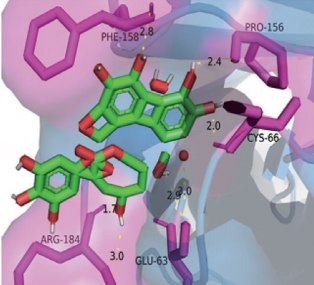

(b)

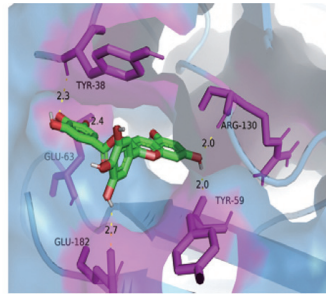

FIGURE 8: Molecular docking diagram. Molecular models of the binding of quercetin, corilagin, geraniin, and EGCG with HSV-2 gD protein, and the results are shown as 3D diagrams. (a) Quercetin-gD. (b) Corilagin-gD. (c) Geraniin-gD. (d) EGCG-gD.
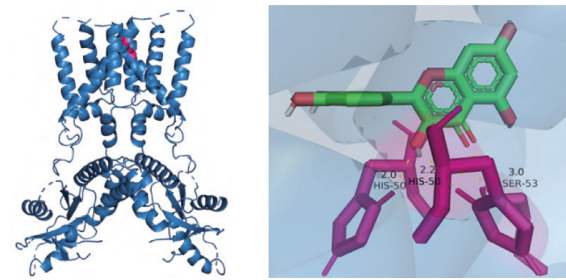

(a)
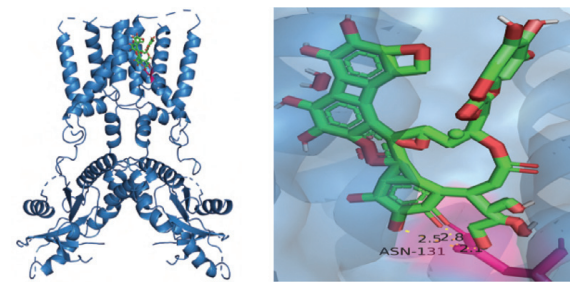

(c)
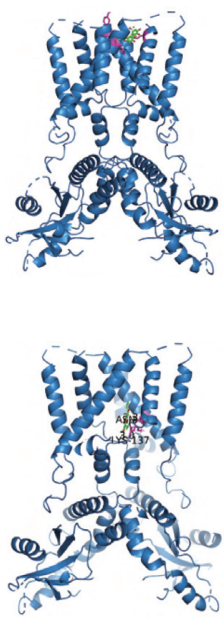

(b)

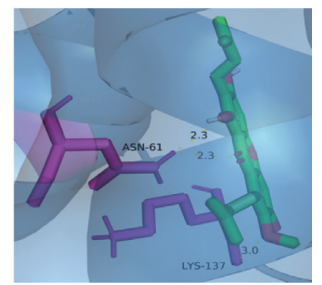

(d)

FIgURE 9: Molecular docking diagram. Molecular models of the binding of quercetin, corilagin, geraniin, and mangostin with STING protein, and the results are shown as 3D diagrams. (a) Quercetin-STING. (b) Corilagin-STING. (c) Geraniin-STING. (d) MangostinSTING.

IL-6 is produced by pathogen-associated molecular patterns (PAMPs) that stimulate cells such as endothelial cells, smooth muscle cells and immune cells to exert a wide range of tissue effects [24]. IL-6 can protect mice from HSV2-induced mortality [25]. Estradiol-treated mice exhibited sooner recruitment and a larger ratio of Th1 and Th17 effector cells in the vagina and better protection after HSV-2 infection compared to placebo-treated controls, and Th17 responses were abolished in IL- $1 \beta$ knockout APC-T cells, suggesting that IL- $1 \beta$ is a crucial element in the induction of Th17 in the reproductive tract [26]. CXCL8, CXCL9, and CXCL10 were found to be expressed at high levels in both HSV-1 and HSV-2 CNS infections in one study [27].
We identified 2 crucial signaling pathways in the IGP network: toll-like receptor (TLR) signaling pathway and cytokine-cytokine receptor interaction pathway. TLR9 pathway specifically recognizes the unmethylated $\mathrm{CpG}$ motifs in dsDNA (CpG DNA) [28]. Studies have confirmed that using pattern recognition receptor (PRR) antagonists, such as lipoproteins, CpG DNA, and cyclic dinucleotides, we can greatly limit HSV-2 replication. TLR9 silencing also affects IL-6 secretion when HSV-2 or viral DNA stimulates the cells [29]. The cytokine-cytokine receptor interaction pathway, according to the annotation of the KEGG (genome.jp/kegg) database, is mainly the interaction between HSV-2 glycoprotein and chemokines such as (CCL26, 
CCL28, CCL22, CCL25, CXCL9, CXCL10, CXCL11, and CXCL13).

Therefore, we found that these signaling pathways are closely associated with HSV-2 infection. GWM may contribute to the therapeutic role of $\mathrm{HSV}-2$ infection by modulating these signaling pathways. The constituents of GWM are highly sophisticated, and since it is impossible to include all of them in the database, some constituents and their targets of action could be overlooked.

\section{Conclusions}

The "multicomponent, multitarget, and multipathway" nature of GWM for HSV-2 infection was demonstrated in this study. Using a network pharmacology approach, we identified that quercetin acts on the targets IL-6, IL- $1 \beta$, and CXCL10 through a key signaling pathway (toll-like receptor signaling pathway and cytokine-cytokine receptor interaction) as a key component in controlling HSV-2 infection. This work offers ideas for future research on the molecular mechanisms of GWM for the treatment of HSV-2 infection, and related studies can be seen in future research.

\section{Data Availability}

All data are available from the corresponding author upon reasonable request.

\section{Conflicts of Interest}

The authors report that there are no conflicts of interest in this work.

\section{Authors' Contributions}

Hao Zhang conceived, designed, and planned the study. Hao Zhang and Ming-Huang Gao acquired and analyzed the data. Hao Zhang, Yang Chen, and Ming-Huang Gao interpreted the results. Hao Zhang drafted the manuscript. Tao Liu contributed to the critical revision of the manuscript. All authors read and approved the final manuscript.

\section{Acknowledgments}

The authors are very grateful for the support of the Wuxi Medical Development Discipline-Infectious Disease Development Discipline Fund (grant number FZXK006).

\section{References}

[1] S. Cole, "Herpes simplex virus," Nursing Clinics of North America, vol. 55, no. 3, pp. 337-345, 2020.

[2] D. V. Desai and S. S. Kulkarni, "Herpes simplex virus: the interplay between HSV, host, and HIV-1," Viral Immunology, vol. 28, no. 10, pp. 546-555, 2015.

[3] J. Hopkins, T. Yadavalli, and R. Suryawanshi, "Vitro and in vivo activity, tolerability, and mechanism of action of BX795 as an antiviral against herpes simplex virus 2 genital infection," Antimicrobial Agents and Chemotherapy, vol. 64, no. 9, 2020 .
[4] D. Liu, Y. Ma, Y. Wang, Z. Su, M. Gu, and J. C. Janson, "Onestep separation and purification of hydrolysable tannins from Geranium wilfordii Maxim by adsorption chromatography on cross-linked 12\% agarose gel," Journal of Separation Science, vol. 34, no. 9, pp. 995-998, 2011.

[5] E. M. D. S. Siqueira, T. L. C. Lima, L. Boff et al., "Antiviral potential of spondias mombin L. Leaves extract against herpes simplex virus type-1 replication using in vitro and in silico approaches," Planta Medica, vol. 86, no. 7, pp. 505-515, 2020.

[6] A. Di Sotto, S. Di Giacomo, and D. Amatore, "A polyphenol rich extract from solanum melongena L. DR2 peel exhibits antioxidant properties and anti-herpes simplex virus type 1 activity in vitro," Molecules, vol. 23, no. 8, 2018.

[7] L.-J. Li, S.-J. Zhang, P. Liu et al., "Corilagin interferes with tolllike receptor 3-mediated immune response in herpes simplex encephalitis," Frontiers in Molecular Neuroscience, vol. 12, p. 83, 2019.

[8] C.-M. Yang, H.-Y. Cheng, T.-C. Lin, L.-C. Chiang, and C.-C. Lin, "The in vitro activity of geraniin and 1,3,4,6-tetraO-galloyl- $\beta$-d-glucose isolated from Phyllanthus urinaria against herpes simplex virus type 1 and type 2 infection," Journal of Ethnopharmacology, vol. 110, no. 3, pp. 555-558, 2007.

[9] Y. Wang, X. Fan, H. Qu, X. Gao, and Y. Cheng, "Strategies and techniques for multi-component drug design from medicinal herbs and traditional Chinese medicine," Current Topics in Medicinal Chemistry, vol. 12, no. 12, pp. 1356-1362, 2012.

[10] D. C. Hao and P. G. Xiao, "Network pharmacology: a Rosetta Stone for traditional Chinese medicine," Drug Development Research, vol. 75, no. 5, pp. 299-312, 2014.

[11] J. Ru, P. Li, J. Wang et al., "TCMSP: a database of systems pharmacology for drug discovery from herbal medicines," Journal of Cheminformatics, vol. 6, no. 1, p. 13, 2014.

[12] H. Liu, J. Wang, W. Zhou, Y. Wang, and L. Yang, "Systems approaches and polypharmacology for drug discovery from herbal medicines: an example using licorice," Journal of Ethnopharmacology, vol. 146, no. 3, pp. 773-793, 2013.

[13] O. Trott and A. J. Olson, "AutoDock Vina: improving the speed and accuracy of docking with a new scoring function, efficient optimization, and multithreading," Journal of Computational Chemistry, vol. 31, no. 2, pp. 455-461, 2010.

[14] R.-K. Kadeppagari, R. L. Sanchez, and T. P. Foster, "HSV-2 inhibits type-I interferon signaling via multiple complementary and compensatory STAT2-associated mechanisms," Virus Research, vol. 167, no. 2, pp. 273-284, 2012.

[15] M. Zhang, Y. Liu, P. Wang et al., "HSV-2 immediate-early protein US1 inhibits IFN- $\beta$ production by suppressing association of IRF-3 with IFN- $\beta$ promoter," The Journal of Immunology, vol. 194, no. 7, pp. 3102-3115, 2015.

[16] X. Guan, M. Zhang, M. Fu, S. Luo, and Q. Hu, "Herpes simplex virus type 2 immediate early protein ICP27 inhibits IFN- $\beta$ production in mucosal epithelial cells by antagonizing IRF3 activation," Frontiers in Immunology, vol. 10, p. 290, 2019.

[17] M. Motwani, S. Pesiridis, and K. A. Fitzgerald, "DNA sensing by the cGAS-STING pathway in health and disease," Nature Reviews Genetics, vol. 20, no. 11, pp. 657-674, 2019.

[18] S. A. Connolly, T. S. Jardetzky, and R. Longnecker, "The structural basis of herpesvirus entry," Nature Reviews Microbiology, vol. 19, no. 2, pp. 110-121, 2021.

[19] C. E. Isaacs, G. Y. Wen, W. Xu et al., "Epigallocatechin gallate inactivates clinical isolates of herpes simplex virus," Antimicrobial Agents and Chemotherapy, vol. 52, no. 3, pp. 962970, 2008. 
[20] M.-M. Hu and H.-B. Shu, "Innate immune response to cytoplasmic DNA: mechanisms and diseases," Annual Review of Immunology, vol. 38, no. 1, pp. 79-98, 2020.

[21] E. I. Tognarelli, T. F. Palomino, N. Corrales, S. M. Bueno, A. M. Kalergis, and P. A. González, "Herpes simplex virus evasion of early host antiviral responses," Frontiers in Cellular and Infection Microbiology, vol. 9, p. 127, 2019.

[22] H. O. Sintim, C. G. Mikek, M. Wang, and M. A. Sooreshjani, "Interrupting cyclic dinucleotide-cGAS-STING axis with small molecules," Medchemcomm, vol. 10, no. 12, pp. 1999-2023, 2019.

[23] J. C. M. Brito, W. G. Lima, L. P. B. Cordeiro, and W. S. da Cruz Nizer, "Effectiveness of supplementation with quercetintype flavonols for treatment of viral lower respiratory tract infections: systematic review and meta-analysis of preclinical studies," Phytotherapy Research, 2021.

[24] A. Y. Liu, "Infectious implications of interleukin-1, interleukin-6, and T helper type 2 inhibition," Infectious Disease Clinics of North America, vol. 34, no. 2, pp. 211-234, 2020.

[25] R. Aoki, T. Kawamura, F. Goshima et al., "Mast cells play a key role in host defense against herpes simplex virus infection through TNF- $\alpha$ and IL-6 production," Journal of Investigative Dermatology, vol. 133, no. 9, pp. 2170-2179, 2013.

[26] V. C. Anipindi, P. Bagri, K. Roth et al., "Estradiol enhances CD4+ T-cell anti-viral immunity by priming vaginal DCs to induce Th17 responses via an IL-1-dependent pathway," PLoS Pathogens, vol. 12, no. 5, p. e1005589, 2016.

[27] L. Lind, M. Studahl, L. Persson Berg, and K. Eriksson, "CXCL11 production in cerebrospinal fluid distinguishes herpes simplex meningitis from herpes simplex encephalitis," Journal of Neuroinflammation, vol. 14, no. 1, p. 134, 2017.

[28] Z. Ma, G. Ni, and B. Damania, "Innate sensing of DNA virus genomes," Annual Review of Virology, vol. 5, no. 1, pp. 341-362, 2018.

[29] K. Triantafilou, D. Eryilmazlar, and M. Triantafilou, "Herpes simplex virus 2 -induced activation in vaginal cells involves Toll-like receptors 2 and 9 and DNA sensors DAI and IFI16," American Journal of Obstetrics and Gynecology, vol. 210, no. 2, pp. 122-e10, 2014. 